\title{
Positive bases in ordered subspaces with the Riesz decomposition property
}

\author{
by \\ Vasilios Katsikis and Ioannis A. Polyrakis (Athens)
}

\begin{abstract}
In this article we suppose that $E$ is an ordered Banach space whose positive cone is defined by a countable family $\mathcal{F}=\left\{f_{i} \mid i \in \mathbb{N}\right\}$ of positive continuous linear functionals on $E$, i.e. $E_{+}=\left\{x \in E \mid f_{i}(x) \geq 0\right.$ for each $\left.i\right\}$, and we study the existence of positive (Schauder) bases in ordered subspaces $X$ of $E$ with the Riesz decomposition property. We consider the elements $x$ of $E$ as sequences $x=\left(f_{i}(x)\right)$ and we develop a process of successive decompositions of a quasi-interior point of $X_{+}$which at each step gives elements with smaller support. As a result we obtain elements of $X_{+}$with minimal support and we prove that they define a positive basis of $X$ which is also unconditional. In the first section we study ordered normed spaces with the Riesz decomposition property.
\end{abstract}

1. Introduction and notations. The most typical examples of ordered Banach spaces $E$ with a rich class of ordered subspaces are the universal spaces $C[0,1]$ and $\ell_{\infty}$. As shown in [8, Theorem 4.1] $\left({ }^{1}\right)$ each separable ordered Banach space with closed and normal positive cone is order-isomorphic to an ordered subspace of $C[0,1]$, therefore the study of positive bases in separable ordered Banach spaces is equivalent to the study of such bases in closed ordered subspaces of $C[0,1]$.

In this article we study the general problem of existence of positive bases in ordered subspaces $X$ of $E$, as formulated in the abstract, by developing a method of decomposition of a quasi-interior point of $X$. To develop this method we study the subspaces $X$ of $E$ with the maximum support property. In such $X$ the quasi-interior points of $X$ and of its closed principal solid subspaces are characterized as the positive vectors of those subspaces with maximum support. We show that in such subspaces the extremal points of $X_{+}$are the nonzero elements of $X_{+}$with minimal support; this property

2000 Mathematics Subject Classification: 46B40, 46B15, 46B42.

Key words and phrases: positive bases, unconditional bases, Riesz decomposition property, quasi-interior points.

We would like to thank an anonymous referee for his helpful remarks and comments.

$\left({ }^{1}\right)$ This result is shown by a slight modification of the classical proof of the universality of $C[0,1]$. 
turns out to be important for the study of positive bases. Also, this class of subspaces is large. Indeed, as shown in [7, Lemma 5.1], each Banach lattice with a positive basis is order isomorphic to a closed, ordered subspace of $\ell_{\infty}$ with the maximum support property with respect to the family $\mathcal{F}$ of the Dirac measures $\delta_{i}$ supported at the natural numbers $i$, and a similar result is also true for the space $C[0,1]$ (see $[8$, Theorem 5.1]). Therefore the class of ordered subspaces of $\ell_{\infty}$ or $C[0,1]$ with the maximum support property is large and contains, in the sense of order isomorphism, the class of Banach lattices with a positive basis.

To develop our method of decompositions we also study the ordered subspaces $X$ of $E$ with the following property which we call the ws-property: for any $x \in X_{+}$and any $f_{i} \in \mathcal{F}$ the set $K=\left\{y \in X_{+} \mid y \leq x\right.$ and $\left.f_{i}(y)=0\right\}$ has at least one maximal element. According to the terminology of vector optimization, $X$ has the ws-property if and only if the set $K$ has Pareto efficient points with respect to $X_{+}$. If $E$ is a Banach lattice with order continuous norm or if $E$ is a dual space, we show, in Corollaries 20 and 21, that the ordered subspaces of $E$ have the ws-property. In the main result of this article, Theorem 32, we prove that the maximum support property and the ws-property are sufficient conditions for the existence of positive bases in the ordered subspaces of $E$ with the Riesz decomposition property. As an application we show (Theorem 36) that the maximum support property and the ws-property are necessary and sufficient for a positive biorthogonal system of an ordered Banach space $E$ with the Riesz decomposition property to define a positive basis of $E$.

This article is a generalization of [7] where the same problem is studied in lattice-subspaces of $E$. In the first section of this paper we study ordered normed spaces with the Riesz decomposition property and we prove some results necessary for our method of decompositions. Specifically we study quasi-interior points and we generalize the results existing for normed lattices to ordered normed spaces with the Riesz decomposition property (Theorems 4 and 6).

Finally, note that each Banach space with an unconditional basis, ordered by the positive cone of the basis, is a Banach lattice with respect to an equivalent norm. The problem of existence of unconditional basic sequences in Banach spaces, known as the unconditional basic sequence problem, was solved in the negative in 1993 by W. T. Gowers and B. Maurey [3]. Our results give necessary conditions for the existence of unconditional basic sequences in ordered Banach spaces.

Let $Y$ be a (partially) ordered normed space with positive cone $Y_{+}$. If $Y=Y_{+}-Y_{+}$then the cone $Y_{+}$is generating or reproducing, and if there exists a real number $a>0$ so that $x, y \in Y_{+}$with $x \leq y$ implies that $\|x\| \leq a\|y\|$, the cone $Y_{+}$is normal. Recall that a convex set $P$ in 
a linear space is a cone if $\lambda x \in P$ for any real $\lambda \geq 0$ and any $x \in P$, and $P \cap(-P)=\{0\}$. For $x, y \in Y$ with $x \leq y$, the set $[x, y]=\{z \in Y \mid x \leq z \leq y\}$ is the order interval $x y$. A point $x \in Y_{+}, x \neq 0$, is an extremal point of $Y_{+}$ if for any $y \in Y$ with $0<y<x$ there exists $\lambda \in \mathbb{R}_{+}$such that $y=\lambda x$.

The space $Y$ has the Riesz decomposition property (RDP) if for any $x, y_{1}, y_{2} \in Y_{+}$with $x \leq y_{1}+y_{2}$ there exist $x_{1}, x_{2} \in Y_{+}$such that $x=x_{1}+x_{2}$ and $0 \leq x_{1} \leq y_{1}, 0 \leq x_{2} \leq y_{2}$. A subspace $Z$ of $Y$ is solid if for any $x, y \in Z$ with $x \leq y$, the order interval $[x, y]$ is contained in $Z$. We say that the cone $Y_{+}$gives an open decomposition of $Y$ or that $Y_{+}$is nonflat if $U_{+}-U_{+}$ is a neighborhood of zero, where $U_{+}=U \cap Y_{+}$is the positive part of the closed unit ball $U$ of $Y$, or equivalently, if any $x \in Y$ has a representation $x=x_{1}-x_{2}$ with $x_{1}, x_{2} \in Y_{+}$and $\left\|x_{1}\right\|,\left\|x_{2}\right\| \leq M\|x\|$, where $M$ is a constant real number.

A linear functional $f$ on $Y$ is positive if $f(x) \geq 0$ for each $x \in Y_{+}$, and strictly positive if $f(x)>0$ for each $x \in Y_{+}, x \neq 0$. Denote by $Y^{*}$ the set of continuous linear functionals of $Y$ and by $Y_{+}^{*}$ the set of positive ones.

Suppose that $Y$ is an ordered Banach space. A sequence $\left\{e_{n}\right\}$ in $Y$ is a (Schauder) basis of $Y$ if each $x \in Y$ has a unique expansion $x=\sum_{n=1}^{\infty} \lambda_{n} e_{n}$ with $\lambda_{n} \in \mathbb{R}$ for each $n$. If moreover $Y_{+}=\left\{x=\sum_{n=1}^{\infty} \lambda_{n} e_{n} \mid \lambda_{n} \geq 0\right.$ for each $n\}$, then $\left\{e_{n}\right\}$ is a positive basis of $Y$. A positive basis is unique in the sense that if $\left\{b_{n}\right\}$ is another positive basis of $Y$, then each element of $\left\{b_{n}\right\}$ is a positive multiple of an element of $\left\{e_{n}\right\}$. If $\left\{e_{n}\right\}$ is a positive basis of $Y$ then, by [9, Theorem 16.3] and [4, Theorems 3.5.2 and 4.1.5], the following statements are equivalent:

(i) the basis $\left\{e_{n}\right\}$ is unconditional,

(ii) the cone $Y_{+}$is generating and normal,

(iii) $Y$ is a Banach lattice with respect to an equivalent norm.

A linear operator $T$ from $Y$ onto an ordered normed space $Z$ is an orderisomorphism of $Y$ onto $Z$ if $T$ is one-to-one, $T$ and $T^{-1}$ are continuous and for each $x \in Y$ we have: $x \in Y_{+}$if and only if $T(x) \in Z_{+}$. For undefined notions and terminology regarding ordered spaces we refer to [4], [5], [1], [6] and [10]. For Schauder bases we refer to [9].

\section{Quasi-interior points in spaces with the Riesz decomposition} property. In this section we denote by $Y$ an ordered normed space with the Riesz decomposition property whose positive cone $Y_{+}$is closed, normal and gives an open decomposition of $Y$. Then, by the Riesz-Kantorovich theorem, the set $Y^{\mathrm{b}}$ of order bounded linear functionals on $Y$ is an order complete linear lattice. For any $x \in Y_{+}$,

$$
I_{x}=\bigcup_{n \in \mathbb{N}}[-n x, n x]
$$


is the solid subspace of $Y$ generated by $x$, and the closure of $I_{x}$ is the closed solid subspace of $Y$ generated by $x$. We prove below that the closure of $I_{x}$ is again solid. Recall the following properties of an ordered Banach space $W$ which we use in this article: (i) If $W_{+}$is closed and generating, then $W_{+}$ gives an open decomposition of $W$ (Krein-Šmulian) and any order bounded linear functional on $W$ is continuous and (ii) the cone $W_{+}$is normal if and only if $W^{*}=W_{+}^{*}-W_{+}^{*}$ (M. Krein); see for example [4, Theorems 3.5.2, 3.5.6 and 3.4.8]. We start with the following obvious result.

Proposition 1. Any solid subspace of $Y$ has the Riesz decomposition property.

Proposition 2. Suppose that $x \in Y_{+}, x \neq 0$ and $I$ is the closure of $I_{x}$. Then:

(i) for any $y \in I_{+}$, there exists an increasing sequence $\left\{y_{n}\right\}$ in $I_{x}$ which converges to $y$, with $0 \leq y_{n} \leq y$ for each $n$,

(ii) $I$ is a solid subspace of $Y$,

(iii) the positive cone $I_{x}^{+}$on $I_{x}$ is generating,

(iv) if we suppose moreover that $Y$ is a Banach space then each positive, continuous, linear functional on I has a positive, continuous, linear extension onto $Y$.

Proof. Let $y \in I_{+}, y \neq 0$. First we shall show that there exists a sequence $\left\{y_{n}^{\prime}\right\}$ in $I_{x} \cap[0, y]$ convergent to $y$. Since $y \in I$, we have $y=\lim _{n \rightarrow \infty} t_{n}$ where $t_{n} \in\left[-\kappa_{n} x, \kappa_{n} x\right]$ and $\left\{\kappa_{n}\right\}$ is an increasing sequence of natural numbers. Hence $t_{n}-y \rightarrow 0$, therefore by [4, Theorem 3.3.5], there exist sequences $\left\{w_{n}\right\}$, $\left\{v_{n}\right\}$ in $Y_{+}$with $t_{n}-y=w_{n}-v_{n}$ and $w_{n}, v_{n} \rightarrow 0$. Then $t_{n}+v_{n}-y=w_{n} \geq 0$, and therefore

$$
y \leq t_{n}+v_{n} \leq \kappa_{n} x+v_{n} .
$$

By the RDP we know that $y=y_{n}^{\prime}+y_{n}^{\prime \prime}$ where $0 \leq y_{n}^{\prime} \leq \kappa_{n} x$ and $0 \leq y_{n}^{\prime \prime} \leq v_{n}$. Since the cone $Y_{+}$is normal and the sequence $v_{n}$ converges to zero, the sequence $y_{n}^{\prime \prime}$ also converges to zero, hence $y_{n}^{\prime} \rightarrow y$, as desired. So for any positive real number $\varepsilon$, we have $\left\|y-y_{n}^{\prime}\right\|<\varepsilon / 2$ for a proper $n$. We put $r_{1}=y_{n}^{\prime}$. Similarly there exists $r_{2} \in I_{x} \cap\left[0, y-r_{1}\right]$ with $\left\|y-r_{1}-r_{2}\right\|<\varepsilon / 2^{2}$ and continuing this process we find a sequence $\left\{r_{n}\right\}$ in $I_{x}$ with $r_{n} \in[0, y-$ $\left.\sum_{i=1}^{n-1} r_{i}\right]$ and $\left\|y-\sum_{i=1}^{n} r_{i}\right\|<\varepsilon / 2^{n}$ for each $n$. Then $y_{n}=\sum_{i=1}^{n} r_{i}$ is an increasing sequence in $[0, y]$ which converges to $y$, proving (i).

For the proof of (ii) it is enough to show that $[0, y] \subseteq I_{+}$for any $y \in I_{+}$. So let $y \in I_{+}$and $z \in[0, y]$. As in the proof of (i) we find again that $y$ satisfies (1) and by the RDP we have $z=z_{n}^{\prime}+z_{n}^{\prime \prime}$ where $0 \leq z_{n}^{\prime} \leq \kappa_{n} x$, $0 \leq z_{n}^{\prime \prime} \leq v_{n}$ and as before $z_{n}^{\prime \prime} \rightarrow 0$. Hence $z_{n}^{\prime} \rightarrow z$, therefore $z \in I$ and statement (ii) follows. 
Statement (iii) is obvious because for any $y \in[-n x, n x]$ we have $0 \leq$ $y+n x \leq 2 n x$, therefore $y+n x=a+b$ where $a, b \in Y_{+}$with $a \leq n x, b \leq n x$, and hence $y=a-(n x-b)$.

Finally, suppose that $f$ is a positive, continuous linear functional on $I$. For any $y \in Y_{+}$we put $L_{y}=\left\{z \in I_{x}^{+} \mid z \leq y\right\}$. Then $L_{y}$ is bounded because the cone $Y_{+}$is normal. For any $y \in Y_{+}$we put $g(y)=\sup \left\{f(z) \mid z \in L_{y}\right\}$. By the RDP and by the fact that $I_{x}$ is solid we have $L_{y}+L_{w}=L_{y+w}$. Therefore $g$ is positively homogeneous and additive on $Y_{+}$. Hence $g$ has a linear and positive extension onto $Y$ which we denote again by $g$, i.e. $g(x)=g\left(x_{1}\right)-g\left(x_{2}\right)$ for any $x=x_{1}-x_{2} \in Y$ with $x_{1}, x_{2} \in Y_{+}$. By [4, Corollary 3.5.6], $g$ is continuous. By the definition of $g$ and by the fact that $I_{x}$ is solid, we have $g(y)=f(y)$ for any $y \in I_{x}^{+}$, therefore $g$ coincides with $f$ on $I_{x}$ because $I_{x}=I_{x}^{+}-I_{x}^{+}$. Since $I_{x}$ is dense in $I$ we conclude that $g$ is also equal to $f$ on $I$, therefore $g$ is an extension of $f$ from $I$ to $Y$.

Definition 3. An element $u \in Z_{+}$of an ordered topological linear space $Z$ is a quasi-interior point of $Z_{+}$(or a quasi-interior positive element of $Z$ ) if the solid subspace $\bigcup_{n \in \mathbb{N}}[-n u, n u]$ of $Z$ generated by $u$ is dense in $Z$.

The above definition extends the notion of the quasi-interior point (see $[1$, p. 259]) from normed lattices to ordered topological linear spaces. It is clear that if $u$ is a quasi-interior point of $Z_{+}$then $f(u)>0$ for any positive, continuous, and nonzero linear functional $f$ on $Z$. In [5, p. 24], the points $u$ of an ordered Banach space $Z$ with $f(u)>0$ for any positive, continuous, nonzero linear functional $f$ on $Z$ are called quasi-interior points of $Z_{+}$. In Theorem 6 we show that in ordered Banach spaces with the RDP, these two definitions are equivalent. By Proposition 2 we get the following result:

TheOREM 4. An element $u \in Y_{+}$is a quasi-interior point of $Y_{+}$if and only if for each $x \in Y_{+}$there exists an increasing sequence $\left\{x_{n}\right\}$ in $I_{u}$ which converges to $x$ with $0 \leq x_{n} \leq x$ for each $n$.

Proposition 5. If $u$ is a quasi-interior point of $Y_{+}$, then $[0, x] \cap[0, u]$ $\neq\{0\}$ for each $x \in Y_{+}, x \neq 0$.

Proof. By the above theorem there exists an increasing sequence $\left\{x_{n}\right\}$ in $I_{u}$ with $0<x_{n} \leq x$ which converges to $x$, therefore the proposition is true.

THEOREM 6. If moreover $Y$ is a Banach space and $u \in Y_{+}$, then the following statements are equivalent:

(i) $u$ is a quasi-interior point of $Y_{+}$,

(ii) $f(u)>0$ for each $f \in Y_{+}^{*}, f \neq 0$. 
Proof. (i) $\Rightarrow$ (ii) is obvious because $f(u)=0$ implies that $f=0$ on $Y$. For the converse suppose that (ii) holds and that the closure $I$ of $I_{u}$ is a proper subspace of $Y$. So there exists $g \in Y^{*}, g \neq 0$, which is identically zero on $I$. Then $|g| \in Y^{*}$ because $Y$ is a Banach space and $|g|$ is positive. It is known that $|g|(y)=\sup g([-y, y])$ for any $y \in Y_{+}$. Since $g \neq 0$ and the positive cone of $Y$ is generating we see that $g(y) \neq 0$ for at least one $y \in Y_{+}$, which implies that $|g| \neq 0$. Therefore $|g|(u)>0$. Since $|g|(u)=\sup g([-u, u])$ it follows that $g$ is not identically zero on the interval $[-u, u]$, a contradiction because $g$ is identically zero on $I$ and $[-u, u] \subseteq I$. Therefore $u$ is a quasi-interior point of $Y_{+}$and (ii) $\Rightarrow$ (i) is proved.

Proposition 7. Let $Z$ be an ordered normed space and suppose that its positive cone $Z_{+}$is complete. Then the following statements are equivalent:

(i) every $y \in Z_{+}, y \neq 0$, is a quasi-interior point of $Z_{+}$,

(ii) $\operatorname{dim} Z=1$.

Proof. Suppose that (i) is true. First we show that the boundary $\vartheta Z_{+}$ of $Z_{+}$is equal to $\{0\}$. By the Bishop-Phelps theorem (see for example [4, Theorem 3.8.14]) the support points of $Z_{+}$are dense in $\vartheta Z_{+}$. Suppose that $r$ is a support point of $Z_{+}$which is supported by the functional $x^{*} \in Z^{*}$, $x^{*} \neq 0$, i.e. $x^{*}(r)=\min \left\{x^{*}(t) \mid t \in Z_{+}\right\}$. Then $x^{*}(r) \leq 0$ because $0 \in Z_{+}$. If we suppose that $x^{*}$ is not positive, there exists $a \in Z_{+}$with $x^{*}(a)<0$. Then $x^{*}$, restricted to the halfline defined by $a$, takes any negative real value, therefore $x^{*}(r)=-\infty$, a contradiction. Hence $x^{*}$ is positive. If we suppose that $r \neq 0$, then $r$ is a quasi-interior point of $Z_{+}$, therefore $x^{*}(r)>0$, a contradiction, because we have found before that $x^{*}(r) \leq 0$, hence $r=0$ and $\vartheta Z_{+}=\{0\}$.

We now show that $Z=Z_{+} \cup\left(-Z_{+}\right)$. So suppose that $w \in Z \backslash Z_{+}$and $y \in Z_{+}, y \neq 0$. Suppose also that $z$ is a point of the line segment $[y, w]$ with $z \in \vartheta Z_{+}$. Then $z=0$, therefore $w \in-Z_{+}$, hence $Z=Z_{+} \cup\left(-Z_{+}\right)$. Suppose now that $w$ is a fixed point of $Z \backslash Z_{+}$. As shown before, for any point $y \in Z_{+}, y \neq 0$, the line segment $[y, w]$ contains 0 , therefore $y$ belongs to the line defined by $w$ and 0 , hence $Z_{+}$is a halfline and $\operatorname{dim} Z=1$. So (i) implies (ii). The converse is clear.

Definition 8. Let $Z$ be an ordered space and $x, y \in Z_{+}$with $x, y \neq 0$. If $[0, x] \cap[0, y]=\{0\}$, we say that $x, y$ are disjoint in $Z_{+}$and write $\inf _{Z_{+}}\{x, y\}=0$.

The next result will be used later for the study of positive bases. Statement (i) is an easy consequence of the Riesz decomposition property.

Proposition 9. Let $Z$ be an ordered normed space with the Riesz decomposition property. Then the following statements are true: 
(i) If the vectors $y_{1}, \ldots, y_{n}$ are pairwise disjoint in $Z_{+}$and $x \in Z_{+}$with $x \leq y_{1}+\cdots+y_{n}$, then:

(a) $x$ has a unique decomposition $x=x_{1}+\cdots+x_{n}$ with $0 \leq x_{i} \leq y_{i}$ for each $i=1, \ldots, n$,

(b) if $x \geq y_{i}$ for each $i=1, \ldots, n$, then $x=y_{1}+\cdots+y_{n}$,

(c) if $\Phi_{1}, \Phi_{2}$ are subsets of $\{1, \ldots, n\}, y_{\Phi_{1}}=\sum_{i \in \Phi_{1}} \lambda_{i} y_{i}, y_{\Phi_{2}}=$ $\sum_{i \in \Phi_{2}} \mu_{i} y_{i}$, where $\lambda_{i}, \mu_{i}$ are positive real numbers and $h \leq y_{\Phi_{1}}$, $h \leq y_{\Phi_{2}}$ then $h$ has a unique decomposition $h=\sum_{i \in \Phi_{1} \cap \Phi_{2}} h_{i}$ where $0 \leq h_{i} \leq \min \left\{\lambda_{i}, \mu_{i}\right\} y_{i}$ for each $i \in \Phi_{1} \cap \Phi_{2}$. If $\Phi_{1} \cap \Phi_{2}=\emptyset$ then $y_{\Phi_{1}}, y_{\Phi_{2}}$ are disjoint in $Z_{+}$.

(ii) If the positive cone $Z_{+}$of $Z$ is normal and the vectors $y_{i}, i \in \mathbb{N}$, are pairwise disjoint in $Z_{+}$, and the sum $\sum_{i=1}^{\infty} y_{i}$ exists, then

(a) $\inf _{Z_{+}}\left\{\sum_{i=1}^{n} y_{i}, \sum_{i=n+1}^{\infty} y_{i}\right\}=0$ for each $n$,

(b) each element $x$ of $Z_{+}$with $0 \leq x \leq \sum_{i=1}^{\infty} y_{i}$ has a unique expansion $x=\sum_{i=1}^{\infty} x_{i}$ with $0 \leq x_{i} \leq y_{i}$ for each $i$.

Proof. The proof of (i) is the following: By the RDP we have $x=$ $x_{1}+\cdots+x_{n}$ with $0 \leq x_{i} \leq y_{i}$ for each $i$. Suppose that also $x=x_{1}^{\prime}+\cdots+x_{n}^{\prime}$ with $0 \leq x_{i}^{\prime} \leq y_{i}$ for each $i$. Then $0 \leq x_{j}^{\prime} \leq x_{1}+\cdots+x_{n}$, therefore $x_{j}^{\prime}=x_{1}^{\prime \prime}+\cdots+x_{n}^{\prime \prime}$ with $0 \leq x_{i}^{\prime \prime} \leq x_{i} \leq y_{i}$ for each $i$, and hence $x_{i}^{\prime \prime}=0$ for each $i \neq j$ because $y_{i}$ and $y_{j}$ are disjoint. So $x_{j}^{\prime} \leq x_{j}$ and similarly $x_{j} \leq x_{j}^{\prime}$, therefore $x_{j}=x_{j}^{\prime}$ for each $j$, and the expansion of $x$ is unique.

If $y_{j} \leq x$ for each $j$, then $y_{j}=y_{j 1}+y_{j 2}+\cdots+y_{j n}$ with $0 \leq y_{j i} \leq x_{i} \leq y_{i}$ for each $i$. But $0 \leq y_{j i} \leq y_{j}$, hence $y_{j i}=0$ for each $i \neq j$. So $y_{j}=y_{j j} \leq$ $x_{j} \leq y_{j}$, therefore $y_{j}=x_{j}$ for each $j$ and (b) is proved.

To prove (c) we remark that $0 \leq h \leq y_{\Phi_{1}}$ implies that $h=\sum_{i \in \Phi_{1}} h_{i}$ with $0 \leq h_{i} \leq \lambda_{i} y_{i}$ for each $i \in \Phi_{1}$. Since $h \leq y_{\Phi_{2}}$ we have $h_{i}=\sum_{j \in \Phi_{2}} h_{i}^{j}$ with $0 \leq h_{i}^{j} \leq \mu_{j} y_{j}$ for any $j \in \Phi_{2}$. Since the vectors $y_{i}$ are disjoint we infer that $h_{i}^{j}=0$ for each $j \neq i$, therefore $h_{i}=h_{i}^{i} \leq \min \left\{\lambda_{i}, \mu_{i}\right\} y_{i}$ and (c) is proved.

To prove statement (a) of (ii) we suppose that $0 \leq h \leq \sum_{i=1}^{n} y_{i}, \sum_{i=n+1}^{\infty} y_{i}$. Then $h=\sum_{i=1}^{n} h_{i}$ with $0 \leq h_{i} \leq y_{i}$ for each $i=1, \ldots, n$. Also $h_{i} \leq$ $y_{n+1}+\sum_{i=n+2}^{\infty} y_{i}$, therefore $h_{i}=h_{n+1}+h_{n+1}^{\prime}$ where $0 \leq h_{n+1} \leq y_{n+1}$ and $0 \leq h_{n+1}^{\prime} \leq \sum_{i=n+2}^{\infty} y_{i}$. Since $y_{i}$ and $y_{n+1}$ are disjoint we deduce that $h_{n+1}=0$, therefore $0 \leq h_{i}=h_{n+1}^{\prime} \leq \sum_{i=n+2}^{\infty} y_{i}$ and by induction $0 \leq$ $h_{i} \leq \sum_{i=n+m}^{\infty} y_{i}$ for each $m \in \mathbb{N}$. Since the cone is normal and the sequence $\sum_{i=n+m}^{\infty} y_{i}$ converges to zero, we have $h_{i}=0$ for each $i=1, \ldots, n$. Therefore $h=0$ and (a) is proved.

To prove (b) suppose that $0 \leq x \leq \sum_{i=1}^{n} y_{i}+\sum_{i=n+1}^{\infty} y_{i}$. Then $x$ has a unique decomposition $x=\sum_{i=1}^{n} x_{i}+x_{n}^{\prime}$ with $0 \leq x_{i} \leq y_{i}$ for each $i=1, \ldots, n$ and $0 \leq x_{n}^{\prime} \leq \sum_{i=n+1}^{\infty} y_{i}$. If we suppose that $m>n$ and $x=\sum_{i=1}^{m} v_{i}+v_{m}^{\prime}$ with $0 \leq v_{i} \leq y_{i}$ for $i=1, \ldots, m$ and $0 \leq v_{m}^{\prime} \leq \sum_{i=m+1}^{\infty} y_{i}$, then $x=$ 
$\sum_{i=1}^{n} v_{i}+\left(\sum_{i=n+1}^{m} v_{i}+v_{m}^{\prime}\right)$, therefore $x_{i}=v_{i}$ for each $i=1, \ldots, n$. Hence the vectors $x_{i}, i \in \mathbb{N}$, are uniquely determined and the expansion $x=\sum_{i=1}^{\infty} x_{i}$ with $0 \leq x_{i} \leq y_{i}$ for each $i$ is unique.

For a further study of the Riesz decomposition property on the space of operators between Banach lattices we refer to [2] and the references therein.

3. Ordered subspaces. In this section we denote by $E$ an infinitedimensional ordered Banach space whose positive cone $E_{+}$is defined by a countable family $\mathcal{F}=\left\{f_{i} \mid i \in \mathbb{N}\right\}$ of positive, continuous linear functionals on $E$, i.e. $E_{+}=\left\{x \in E \mid f_{i}(x) \geq 0\right.$ for each $\left.i\right\}$. Also we denote by $X$ an ordered subspace of $E$, i.e. $X$ is a subspace of $E$ ordered by the induced ordering. It is clear that $E_{+}$is closed and that $X_{+}=X \cap E_{+}$is the positive cone of $X$. For any $x, y \in X$, denote by $\sup _{X}\{x, y\}$ the supremum and by $\inf _{X}\{x, y\}$ the infimum of $\{x, y\}$ in $X$ whenever they exist. If $\sup _{X}\{x, y\}$ and $\inf _{X}\{x, y\}$ exist for any $x, y \in X$, we say that $X$ is a lattice-subspace of $E$. According to our notations, for any $x, y \in X$ with $x \leq y$ the set $[x, y]_{X}=\{z \in X \mid x \leq z \leq y\}$ is the order interval $x y$ in $X$; if $x, y \in X_{+}$ with $[0, x]_{X} \cap[0, y]_{X}=\{0\}$, we say that $x, y$ are disjoint in $X_{+}$and we write $\inf _{X_{+}}\{x, y\}=0$. Also for any $x \in X_{+}, x \neq 0$, we denote by $I_{x}(X)=$ $\bigcup_{n=1}^{\infty}[-n x, n x]_{X}$ the solid subspace of $X$ generated by $x$. The closure $\overline{I_{x}(X)}$ of $I_{x}(X)$ in $X$ is the closed solid subspace of $X$ generated by $x$. If $\overline{I_{x}(X)}=X$, then $x$ is a quasi-interior point of $X_{+}$.

3.1. The minimal and maximum support properties. The minimal and maximum support properties have been introduced in [7]. For any point $x \in E$ we denote by $x(i)$ the real number $f_{i}(x)$ and by $\operatorname{supp}(x)=\{i \in \mathbb{N} \mid$ $x(i) \neq 0\}$ the support of $x$ (with respect to $\mathcal{F}$ ). The set $\operatorname{supp}\left(X_{+}\right)=$ $\bigcup_{x \in X_{+}} \operatorname{supp}(x)$ is the support of $X_{+}$(with respect to $\left.\mathcal{F}\right)$. An element $x \neq 0$ of $X_{+}$has minimal support in $X_{+}$(with respect to $\mathcal{F}$ ) if for any $y \in X_{+}$, $\operatorname{supp}(y) \subsetneq \operatorname{supp}(x)$ implies $y=0$.

Definition 10. The ordered subspace $X$ of $E$ has the minimal support property (with respect to $\mathcal{F}$ ) if for each $x \in X_{+} \backslash\{0\}$ we have: $x$ is an extremal point of $X_{+}$if and only if $x$ has minimal support in $X_{+}$.

Proposition 11. Suppose $I$ is the closed solid subspace of $X$ generated by a nonzero, positive element $x$ of $X_{+}$. Then $\operatorname{supp}(u)=\operatorname{supp}\left(I_{+}\right)$for any quasi-interior point $u$ of $I_{+}$. (The converse is not always true.)

Proof. It is clear that $\operatorname{supp}(u) \subseteq \operatorname{supp}\left(I_{+}\right)$. If $f_{i}(u)=0$ for some $i \in$ $\operatorname{supp}\left(I_{+}\right)$, then $f_{i}$ is identically zero on $I_{u}(X)$ and therefore also on $I$, a contradiction because we have supposed that $i \in \operatorname{supp}\left(I_{+}\right)$. Hence $f_{i}(u)>0$ and $\operatorname{supp}(u)=\operatorname{supp}\left(I_{+}\right)$. By Example 15(ii) below, the converse is not always true. 
Definition 12. The ordered subspace $X$ of $E$ has the maximum support property (with respect to $\mathcal{F}$ ) if each subspace $F$ of $X$ which is either $X$ or a closed solid subspace of $X$ generated by a nonzero element of $X_{+}$has the property: an element $x \in F_{+}$is a quasi-interior point of $F_{+}$if and only if $\operatorname{supp}(x)=\operatorname{supp}\left(F_{+}\right)$.

Proposition 13. If $X_{+}$is closed and $X$ has the maximum support property, then $X_{+}$has quasi-interior points.

Proof. For each $i \in \operatorname{supp}\left(X_{+}\right)$there exists $x_{i} \in X_{+}$with $f_{i}\left(x_{i}\right)>0$. So

$$
u=\sum_{i \in \operatorname{supp}\left(X_{+}\right)} \frac{x_{i}}{2^{i}\left\|x_{i}\right\|}
$$

is a quasi-interior point of $X_{+}$because $X$ has the maximum support property and $\operatorname{supp}(u)=\operatorname{supp}\left(X_{+}\right)$.

The proof of the next proposition is the same as that of Proposition 3.4 of [7]. The extra assumption here that $X_{+}$is closed is made in order to be able to use Proposition 7.

Proposition 14. If $X_{+}$is closed and $X$ has the maximum support property, then $X$ has the minimal support property.

EXAMPLE 15. (i) The sequence spaces $c_{0}$ and $\ell_{p}$ for $1 \leq p<\infty$ have the maximum support property with respect to the family $\mathcal{F}=\left\{\delta_{i}\right\}$ of Dirac measures $\delta_{i}(x)=x(i)$ supported at the natural numbers $i$. The space $\ell_{\infty}$ of bounded real sequences does not have the maximum support property with respect to $\mathcal{F}$. Indeed, the vector $x$ with $x(i)=1 / i$ for any $i$ has maximum support and the closed solid subspace generated by $x$ is $c_{0}$. On the other hand, $\ell_{\infty}$ has the minimal support property because the extremal points of $\ell_{\infty}^{+}$, being positive multiples of the vectors $e_{i}$, have minimal support.

(ii) The family $\left\{\delta_{r_{i}} \mid i \in \mathbb{N}\right\}$ of Dirac measures supported at the rational numbers $r_{i}$ in $[0,1]$ and also the family $\mathcal{G}=\left\{\mu_{i} \mid i \in \mathbb{N}\right\}$ of Lebesgue measures $\mu_{i}$ restricted to $I_{i}$, where $\left\{I_{i}\right\}$ is a sequence of subintervals of $[0,1]$ so that each interval $(a, b)$ of $[0,1]$ contains at least one $I_{i}$, define the positive cone of the space $E=C[0,1]$ of continuous, real-valued functions defined on $[0,1]$. The space $E$ does not have the maximum support property with respect to these families. Indeed, if $x \in E_{+}$with $x\left(t_{0}\right)=0$ for some irrational number $t_{0}$ and $x(t)>0$ for each $t \neq t_{0}$, then $\operatorname{supp}(x)=\mathbb{N}$ but $x$ is not a quasi-interior point of $E_{+}$.

Theorem 16 ([8, Proposition 2.5]). If $X$ is closed and $X$ has a positive basis $\left\{b_{n}\right\}$, the following statements are equivalent:

(i) $X$ has the maximum support property with respect to $\mathcal{F}$, 
(ii) there exists a sequence $\left\{i_{n}\right\}$ in $\mathbb{N}$ such that $f_{i_{n}}\left(b_{n}\right)>0$ and $f_{i_{n}}\left(b_{m}\right)=0$ for any $m \neq n$, i.e. the coefficient functionals of the basis $\left\{b_{n}\right\}$ can be extended onto $E$ to positive multiples of elements of $\mathcal{F}$.

Here is an example of an ordered subspace with a positive basis, but without the maximum support property.

EXAMPLE 17. Let $\left\{b_{n}\right\}$ be a sequence in $\ell_{\infty}$ so that $b_{1}(4 n)=1 / 2^{n}$, $b_{1}(4 n+1)=1 / 3^{n}$ and $b_{1}(i)=0$ in the other cases, $b_{2}(4 n)=1 / 3^{n}$, $b_{2}(4 n+1)=1 / 2^{n}$ and $b_{2}(i)=0$ in the other cases, and $b_{n}=e_{4 n+2}$ for $n \geq 3$. Then $\left\{b_{n}\right\}$ is a positive basis of the closed subspace $X$ of $\ell_{\infty}$ generated by it. The subspace $X$ does not have the maximum support property with respect to the family $\mathcal{F}$ of Dirac measures $\delta_{i}$ supported at the natural numbers $i$. Indeed, $\operatorname{supp}\left(b_{1}\right)=\operatorname{supp}\left(b_{2}\right)$, therefore $\delta_{i}\left(b_{1}\right)>0$ if and only if $\delta_{i}\left(b_{2}\right)>0$, and by Theorem $16, X$ does not have the maximum support property.

3.2. The ws-property. The notion of the s-property (supremum property) has been introduced in [7]. We define here a weaker property, which we call the ws-property (weak s-property), as follows:

Definition 18. An ordered subspace $X$ of $E$ has the ws-property (with respect to $\mathcal{F})$ if for each $x \in X_{+}, x \neq 0$, and for each $i \in \operatorname{supp}\left(X_{+}\right)$the set $\left\{y \in[0, x]_{X} \mid y(i)=0\right\}$ has at least one maximal element.

If in the above definition the set $\left\{y \in[0, x]_{X} \mid y(i)=0\right\}$ has a maximum element, then $X$ has the s-property. If $X$ has the ws-property, each solid subspace $Z$ of $X$ has this property. In the theory of vector optimization the maximal elements of a subset $K$ of a normed space $Z$ with respect to an ordering cone $P$ of $Z$ are the Pareto efficient points of $K$. In our case, the ws-property ensures the existence of Pareto efficient points with respect to $X_{+}$. We start with the following easy result.

TheOrem 19. Suppose that $\tau$ is a linear topology on $E$ and

(i) $X_{+}$is $\tau$-closed,

(ii) each increasing net in $X_{+}$, order bounded in $X$, has a $\tau$-convergent subnet,

(iii) for each $i$ the positive part $K_{i}^{+}=\left\{y \in X_{+} \mid f_{i}(y)=0\right\}$ of the kernel of $f_{i}$ in $X$ is $\tau$-closed.

Then $X$ has the ws-property.

Proof. Suppose that $x \in X_{+}$and that $A$ is a totally ordered subset of the $\tau$-closed set $[0, x]_{X} \cap K_{i}^{+}$. For each finite subset $\Phi$ of $A$ denote by $x_{\Phi}$ the maximum of $\Phi$. Then $\left\{x_{\Phi}\right\}$, being an increasing, order bounded net in $[0, x]_{X} \cap K_{i}^{+}$, is convergent to $x_{0} \in[0, x]_{X} \cap K_{i}^{+}$which is an upper bound of $A$, and by Zorn's lemma the set $[0, x]_{X} \cap K_{i}^{+}$has maximal elements. 
Corollary 20. If $E$ is a Banach lattice with order continuous norm and $X_{+}$is closed, then $X$ has the ws-property.

Proof. Each order interval in $E$ is weakly compact. Since $X_{+}$is weakly closed, each order interval in $X$ is weakly compact, hence $X$ has the wsproperty.

Corollary 21. If $E$ is a dual space, the functionals $f_{i}$ are weak-star continuous and $X_{+}$is weak-star closed and normal, then $X$ has the wsproperty.

Proof. For each $x \in X_{+}$the order interval $[0, x]_{X}$ is weak-star closed and bounded because $X_{+}$is normal, therefore $[0, x]_{X}$ is weak-star compact. Hence $X$ has the ws-property.

Corollary 22. If $X$ is closed with a positive basis, then $X$ has the ws-property.

Proof. By [11, Theorem 5], each order interval of $X$ is compact.

ExAmPle 23. (i) The spaces $c_{0}$ and $\ell_{p}$ with $1 \leq p<\infty$ and also the spaces $L_{p}^{+}(\mu), 1 \leq p<\infty$, being Banach lattices with order continuous norm, have the ws-property with respect to any countable family which defines their positive cone. Also all their closed ordered subspaces have the ws-property.

(ii) By Corollary 21, $\ell_{\infty}$ and its weak-star closed ordered subspaces have the ws-property with respect to the family of Dirac measures supported at natural numbers.

(iii) $C[0,1]$ does not have the ws-property with respect to the family of Dirac measures supported at rational numbers in $[0,1]$. It is easy to show that the set $\{y \in C[0,1] \mid 0 \leq y \leq x$ and $y(1 / 2)=0\}$, where $x \in C_{+}[0,1]$ with $x(1 / 2)>0$, does not have maximal elements.

If $P, Q, R$ are subcones of $X_{+}$with $R=P+Q$ and $P \cap Q=\{0\}$, we say that $R$ is the direct sum of $P, Q$ and write $P \oplus Q=R$.

Proposition 24. Suppose that $X$ is closed, $X_{+}$is generating and normal, and $X$ has the Riesz decomposition property and the ws-property with respect to $\mathcal{F}$. Let $x \in X_{+}, x \neq 0, i \in \operatorname{supp}\left(X_{+}\right)$and denote by $z_{i}$ a maximal element of $\left\{y \in[0, x]_{X} \mid y(i)=0\right\}$. Then $z_{i}^{\prime}=x-z_{i}$ is a minimal element of $\left\{y \in[0, x]_{X} \mid y(i)=x(i)\right\}$. If $I, J, W$ are the closed solid subspaces of $X$ generated respectively by $x, z_{i}, z_{i}^{\prime}$, then:

(i) $\inf _{X_{+}}\left\{z_{i}, z_{i}^{\prime}\right\}=0$.

(ii) The functional $f_{i}$ is identically zero on $J$. If $f_{i}(x)>0$ then $f_{i}$ is strictly positive on $W$. If $f_{i}(x)=0$, then $z_{i}=x$, and if $f_{i}$ is 
strictly positive on $I$, then $z_{i}^{\prime}=x$. If $f_{i}$ is not identically zero and non-strictly positive on $I$ then $0<z_{i}<x$ and $0<z_{i}^{\prime}<x$.

(iii) If $f_{i}(x)>0$, then $I_{z_{i}}^{+}(X) \oplus I_{z_{i}^{\prime}}^{+}(X)=I_{x}^{+}(X)$ and $J_{+} \oplus W_{+}=I_{+}$.

Proof. Suppose that $z \in A=\left\{y \in[0, x]_{X} \mid y(i)=x(i)\right\}$ with $z_{i}^{\prime}>z$. Then $x-z>z_{i}$ and $f_{i}(x-z)=0$, which contradicts the definition of $z_{i}$. Therefore $z_{i}^{\prime}$ is a minimal element of $A$.

(i) Let $h \in X$ with $0<h \leq z_{i}, z_{i}^{\prime}$. Then $0 \leq h(i) \leq z_{i}(i)=0$, hence $h(i)=0$. So $h+z_{i} \leq x$ and $\left(h+z_{i}\right)(i)=0$, a contradiction. It follows that $\inf _{X_{+}}\left\{z_{i}, z_{i}^{\prime}\right\}=0$.

(ii) Since $z_{i}(i)=0, f_{i}$ is identically zero on $I_{z_{i}}$ and therefore also on $J$. Suppose that $f_{i}(x)>0$. Then $z_{i}<x$, hence $z_{i}^{\prime}>0$ and $W_{+} \neq\{0\}$. Suppose that $w \in W_{+}, w>0$ and $w(i)=0$. Then by Theorem $4, w$ is the limit of an increasing sequence of elements of $I_{z_{i}^{\prime}}^{+}(X)$, therefore $y(i)=0$ for at least one $y \in X$ with $0<y \leq z_{i}^{\prime}$. Then $y+z_{i} \leq x$ and $\left(y+z_{i}\right)(i)=0$, a contradiction, therefore $f_{i}$ is strictly positive on $W$. If we suppose that $f_{i}(x)=0$, then by the definition of $z_{i}$ we have $z_{i}=x$ and if $f_{i}$ is strictly positive on $I$ then $z_{i}=0$, therefore $z_{i}^{\prime}=x$. Suppose now that $f_{i}$ is nonzero and also non-strictly positive on $I$. Then $x(i)>0$ and also $v(i)=0$ for at least one nonzero point $v$ of $I_{+}$. Since $v$ is the limit of an increasing sequence of elements of $I_{x}^{+}(X)$, we have $y(i)=0$ for at least one nonzero $y \in[0, x]_{X}$. This implies that $z_{i}>0$ because if $z_{i}=0$ then $z_{i}<y$, which contradicts the definition of $z_{i}$. Also $z_{i}<x$ because $x(i)>0$. So $0<z_{i}<x$ and $0<z_{i}^{\prime}<x$.

(iii) Let $f_{i}(x)>0$. Suppose that $h \in J_{+} \cap W_{+}$. Then $h \in J_{+}$and therefore $h(i)=0$. Since $f_{i}$ is strictly positive on $W$ we have $h=0$, therefore $J_{+} \cap W_{+}=\{0\}$. Suppose that $y \in[0, x]_{X}$. Then $y \leq z_{i}+z_{i}^{\prime}$ and by the RDP we have $y=y_{1}+y_{2}$ with $y_{1} \in\left[0, z_{i}\right]_{X}$ and $y_{2} \in\left[0, z_{i}^{\prime}\right]_{X}$. By the above remarks the first assertion of (iii) is proved.

Suppose now that $y \in I_{+}$. By Theorem $4, y$ is the limit of an increasing sequence $y_{n}$ in $I_{x}^{+}(X)$ with $y_{n} \leq y$ for each $n$. Hence $y_{n+1}-y_{n} \in I_{x}^{+}(X)$, therefore $y_{n+1}-y_{n} \leq k_{n} x=k_{n}\left(z_{i}+z_{i}^{\prime}\right)$, and by the RDP we have $y_{n+1}-y_{n}=$ $a_{n+1}+b_{n+1}$ with $a_{n+1} \in I_{z_{i}}^{+}(X)$ and $b_{n+1} \in I_{z_{i}^{\prime}}^{+}(X)$. If $y_{1}=a_{1}+b_{1}$ with $a_{1} \in I_{z_{i}}^{+}(X)$ and $b_{1} \in I_{z_{i}^{\prime}}^{+}(X)$, then

$$
y_{n}=\left(a_{1}+\cdots+a_{n}\right)+\left(b_{1}+\cdots+b_{n}\right) .
$$

If $s_{n}=a_{1}+\cdots+a_{n}$ and $r_{n}=b_{1}+\cdots+b_{n}$, then $s_{n+1}-s_{n}=a_{n+1} \leq y_{n+1}-y_{n}$, therefore the sequence $\left\{s_{n}\right\}$ is convergent, because $\left\{y_{n}\right\}$ is convergent and the cone $X_{+}$is normal. Similarly, $\left\{r_{n}\right\}$ is convergent and therefore $y=y^{\prime}+y^{\prime \prime}$ with $y^{\prime} \in J_{+}$and $y^{\prime \prime} \in W_{+}$. Hence $I_{+}=J_{+} \oplus W_{+}$.

Definition 25. Let $X$ be a closed ordered subspace of $E$ as in the previous proposition, and suppose that $x$ is a nonzero element of $X_{+}$and $f_{i} \in \mathcal{F}$. If $f_{i}$ is not identically zero and non-strictly positive on $I_{x}(X)$ and 
$x=x_{1}+x_{2}$ where $x_{1}$ is a maximal element of the set $\left\{y \in[0, x]_{X} \mid y(i)=0\right\}$, then we say that $x=x_{1}+x_{2}$ is a decomposition of $x$ with respect to $f_{i}$ (or with respect to $i$ ) and also that $x$ is decomposable into $x_{1}, x_{2}$ with respect to $f_{i}$. If $f_{i}$ is identically zero on $I_{x}(X)$ or if $f_{i}$ is strictly positive on $I_{x}(X)$, we say that $x$ is indecomposable with respect to $f_{i}$ (or with respect to $i$ ).

3.3. Existence of positive bases. In what follows we will denote by $X$ a closed, ordered subspace of $E$ so that:

(i) $X$ has the Riesz decomposition property,

(ii) the positive cone $X_{+}$of $X$ is closed, normal and generating,

(iii) $X$ has the maximum support property and the ws-property with respect to $\mathcal{F}$.

As noted at the beginning of the previous section, (i) and (ii) imply that $X_{+}$gives an open decomposition of $X$ and that $X^{*}$ is an order complete linear lattice. We will also denote by $M$ the following subset of $\mathbb{N}$ :

$$
M=\left\{i \in \operatorname{supp}\left(X_{+}\right) \mid f_{i} \text { is non-strictly positive on } X\right\} .
$$

Therefore for each $x \in X_{+}, x \neq 0$, we have $x(i)>0$ for each $i \in$ $\operatorname{supp}\left(X_{+}\right) \backslash M$. Also $M \neq \emptyset$ because $M=\emptyset$ implies $\operatorname{supp}(x)=\operatorname{supp}\left(X_{+}\right)$ for each $x \in X_{+}, x \neq 0$, therefore $\operatorname{dim} X=1$ by Proposition 7 . In order to prove the existence of extremal points of $X_{+}$we develop a process of successive decompositions of a quasi-interior point of $X_{+}$. So suppose that $u$ is a quasi-interior point of $X_{+}$(such a point exists by Proposition 13); we decompose $u$ as follows:

SteP 1. We put $i_{1}=\min M$ and we decompose $u$ into $x_{1}, x_{2}$ with respect to $i_{1}$. Then $u=x_{1}+x_{2}$ and $\inf _{X_{+}}\left\{x_{1}, x_{2}\right\}=0$. Also $f_{i_{1}}$ is identically zero on $I_{1}$ and strictly positive on $I_{2}$ where $I_{1}, I_{2}$ are the closed solid subspaces of $X$ generated by $x_{1}, x_{2}$ respectively. The set $m_{1}=\left\{x_{1}, x_{2}\right\}$ is the front and the natural number $i_{1}$ is the index of the first decomposition.

STEP $\nu+1$. Suppose that we have accomplished the $\nu$ th step and that $m_{\nu}$ is the front and $i_{\nu}$ the index of the $\nu$ th decomposition. Then at least one of the elements of $m_{\nu}$ is decomposable with respect to an $i \in M$. Indeed, if no element $x$ of $m_{\nu}$ is decomposable with respect to any $i \in M$ then for any $i \in M, f_{i}$ is strictly positive or identically zero on the closed solid subspace $I$ of $X$ generated by $x$ and it is easy to show that $\operatorname{supp}(y)=\operatorname{supp}\left(I_{+}\right)$for any $y \in I_{+}, y \neq 0$, so $y$ is a quasi-interior point of $I$. Hence $\operatorname{dim} I=1$ and $X$ is finite-dimensional because $m_{\nu}$ is finite. Put $i_{\nu+1}=\min \{i \in M \mid$ at least one element of $m_{\nu}$ is decomposable with respect to $\left.i\right\}$. Then $i_{\nu+1}>i_{\nu}$ and we decompose with respect to $i_{\nu+1}$ the elements of $m_{\nu}$ which allow such a decomposition. We denote by $m_{\nu+1}$ the set which contains the elements of $m_{\nu}$ which are indecomposable with respect to $i_{\nu+1}$ and also the elements that 
arise from the decomposition of the elements of $m_{\nu}$ with respect to $i_{\nu+1}$. The set $m_{\nu+1}$ is the front and $i_{\nu+1}$ is the index of the $(\nu+1)$ th decomposition. The set

$$
\delta(u)=\bigcup_{\nu=0}^{\infty} m_{\nu},
$$

where $m_{0}=\{u\}$, is the tree of decompositions of $u$.

Proposition 26. In the above process of decompositions of $u$ we have:

(i) the sequence $\left\{i_{\nu}\right\}$ of indices of decompositions is strictly increasing,

(ii) for each $i \in M$ with $i \leq i_{\nu}$ and for each $x \in m_{\nu}, x$ is indecomposable with respect to $i$, so $f_{i}$ is strictly positive or identically zero on $I=\overline{I_{x}(X)}$

(iii) the elements of $m_{\nu}$ are nonzero with sum $u$. Also $\inf _{X_{+}}\{x, y\}=0$ for any $x, y \in m_{\nu}$ with $x \neq y$,

(iv) $\inf _{X_{+}}\{x, u-x\}=0$ for each $x \in \delta(u)$.

Proof. Statements (i)-(iii) are obvious. To prove (iv) we suppose that $x \in m_{\nu}$ for some $\nu$ and that $m_{\nu}=\left\{x, y_{1}, \ldots, y_{k}\right\}$. Since the elements of $m_{\nu}$ are pairwise disjoint in $X_{+}$with sum $u$ we have $u-x=\sum_{i=1}^{k} y_{i}$ and (iv) is true by Proposition 9 .

For any $x \in m_{\nu}$ with $\nu \geq 1$ it is easy to show that there exists a unique vector $y \in m_{\nu-1}$ with $y \geq x$. Also for any $x \in m_{\nu}$ there exists at least one $y \in m_{\nu+1}$ with $x \geq y$. If $x, y \in \delta(u)$ with $x \in m_{\nu}, y \in m_{\nu+\mu}$ and $y \leq x$, we say that $x$ is the presuccessor of $y$ in $m_{\nu}$, or that $y$ is a successor of $x$ in $m_{\nu+\mu}$. If moreover $y \in m_{\nu+1}$ we say that $x$ is the first presuccessor of $y$ or that $y$ is a first successor of $x$.

Proposition 27. The following are true:

(i) for any $x \in m_{\nu}$ the sum of the successors of $x$ in $m_{\nu+\mu}$ is equal to $x$,

(ii) if $y$ is a successor of $x$ with $x>y$ and $I$ is the closed solid subspace of $X$ generated by $x$, then $\inf _{X_{+}}\{y, x-y\}=0$ and $y$ is not a quasiinterior point of $I_{+}$,

(iii) for each $x \in \delta(u)$ and each $i \in M \cap \operatorname{supp}(x)$, there exists a successor $y$ of $x$ such that the functional $f_{i}$ is strictly positive on the closed solid subspace $I$ of $X$ generated by $y$.

Proof. (i) Any element of $\delta(u)$ is the sum of its first successors, therefore the proposition is true for $\mu=1$ and continuing, we deduce it for any $\mu$.

(ii) Since $x-y \leq u-y$ and $\inf _{X_{+}}\{y, u-y\}=0$ we have $\inf _{X_{+}}\{y, x-y\}$ $=0$, therefore $y$ is not a quasi-interior point of $I_{+}$by Proposition 5 .

(iii) Let $x \in m_{\kappa}$. Since the sequence $\left\{i_{\nu}\right\}$ is strictly increasing, there exists $\nu \in \mathbb{N}$ with $\nu>\kappa$ and $i \leq i_{\nu}$. Then $f_{i}$ is strictly positive or identically 
zero on any closed solid subspace of $X$ generated by an element of $m_{\nu}$. But $x=\sum_{j=1}^{r} x_{j}$ where $x_{1}, \ldots, x_{r}$ are the successors of $x$ in $m_{\nu}$ and $f_{i}(x)>0$ because $i \in \operatorname{supp}(x)$, therefore $f_{i}$ is strictly positive on at least one of the closed solid subspaces of $X$ generated by $x_{1}, \ldots, x_{r}$, which proves (iii).

If $x \in \delta(u)$ and $x \in m_{\nu}$ for each $\nu \geq \nu_{0}$, then we will say that the process of decomposition stops at $x$. In other words, the process of decomposition stops at $x$ if there exists $\nu_{0} \in \mathbb{N}$ so that $x \in m_{\nu_{0}}$ and for each $i \in M$ with $i>i_{\nu_{0}}$, the functional $f_{i}$ is strictly positive or identically zero on the closed solid subspace $I$ of $X$ generated by $x$. Then for each $i \in M$ with $i \leq i_{\nu_{0}}$, $f_{i}$ is strictly positive or identically zero on $I$ (Proposition 26), therefore $\operatorname{supp}(z)=\operatorname{supp}\left(I_{+}\right)$for any $z \in I_{+}, z \neq 0$, hence any nonzero vector of $I_{+}$is a quasi-interior point of $I$, which implies that $\operatorname{dim} I=1$. So $x$ is an extremal point of $X_{+}$and we have proved the following:

Proposition 28. If the process of decompositions of $u$ stops at an element $x_{0} \in \delta(u)$ then $x_{0}$ is an extremal point of $X_{+}$.

A sequence $\left\{x_{\nu}\right\}$ in $\delta(u)$ is a branch of $\delta(u)$ if $x_{\nu}>x_{\nu+1}$ for each $\nu \in \mathbb{N}$.

Proposition 29. Each branch of $\delta(u)$ converges to zero.

Proof. It is enough to show that any branch $\left\{x_{\nu}\right\}$ of $\delta(u)$ with $x_{0}=u$ converges to zero. Let $z_{\nu}=x_{\nu-1}-x_{\nu}$ for all $\nu \geq 1$. Then for all $\nu, \mu$, we have

$$
u=z_{1}+\cdots+z_{\nu}+x_{\nu} \quad \text { and } \quad x_{\nu}=z_{\nu+1}+\cdots+z_{\nu+\mu}+x_{\nu+\mu} .
$$

The vectors $z_{1}, \ldots, z_{\nu}, x_{\nu}$ are pairwise disjoint in $X_{+}$. Indeed, we have $\inf _{X_{+}}\left\{x_{\nu}, u-x_{\nu}\right\}=0$, hence $\inf _{X_{+}}\left\{x_{\nu}, \sum_{i=1}^{\nu} z_{i}\right\}=0$, therefore inf $X_{+}\left\{x_{\nu}, z_{i}\right\}$ $=0$ for each $i \leq \nu$, because $z_{i} \leq \sum_{j=1}^{\nu} z_{j}$. Suppose that $j>i$. Then $z_{j} \leq x_{i}$ and $\inf _{X_{+}}\left\{z_{i}, x_{i}\right\}=0$, therefore $\inf _{X_{+}}\left\{z_{j}, z_{i}\right\}=0$. Hence $\inf _{X_{+}}\left\{z_{j}, z_{i}\right\}=0$ for any $i \neq j$.

Let $u_{0}=\sum_{\nu=1}^{\infty} z_{\nu} / 2^{\nu}$. We shall show that $\operatorname{supp}\left(u_{0}\right)=\operatorname{supp}\left(X_{+}\right)$. For each $i \in \operatorname{supp}\left(X_{+}\right) \backslash M$ we have $x(i)>0$ for each $x \in X_{+}, x \neq 0$, hence $i \in \operatorname{supp}\left(u_{0}\right)$. Suppose that $i \in M$ and that $x_{\nu}$ is decomposed at the $\kappa_{\nu}$ th decomposition. Since $\left\{i_{\kappa_{\nu}}\right\}$ is strictly increasing, there exists $\mu \in \mathbb{N}$ with $i<i_{\kappa_{\mu}}$. By Proposition 26(ii), $f_{i}$ is strictly positive or identically zero on $I=\overline{I_{x_{\mu}}(X)}$. We shall show that in both cases $i \in \operatorname{supp}\left(u_{0}\right)$. If $f_{i}$ is strictly positive on $I$ we have $z_{\mu+1}(i)>0$ because $0<z_{\mu+1}<x_{\mu}$, and therefore $i \in \operatorname{supp}\left(u_{0}\right)$. If $f_{i}$ is identically zero on $I$ then $x_{\mu}(i)=0$, therefore

$$
f_{i}\left(z_{1}+\cdots+z_{\mu}\right)=f_{i}\left(z_{1}+\cdots+z_{\mu}+x_{\mu}\right)=f_{i}(u)>0,
$$

hence $f_{i}\left(z_{j}\right)>0$ for at least one $j$, so $i \in \operatorname{supp}\left(u_{0}\right)$. Therefore $\operatorname{supp}\left(X_{+}\right)=$ $\operatorname{supp}\left(u_{0}\right)$ and $u_{0}$ is a quasi-interior point of $X$.

By Theorem 4, there is an increasing sequence $\phi_{n} \in[0, u]_{X} \cap\left[0, r_{n} u_{0}\right]_{X}$, where $\left\{r_{n}\right\}$ is a strictly increasing sequence of natural numbers with 
$\lim _{n \rightarrow \infty} \phi_{n}=u$. Let

$$
h_{\nu}=\sum_{\mu=1}^{\infty} r_{\nu} \frac{z_{r_{\nu}+\mu}}{2^{r_{\nu}+\mu}} .
$$

Since $0 \leq \phi_{n} \leq r_{n} u_{0}$ we have $0 \leq \phi_{n} \leq r_{n} z_{1}+\cdots+r_{n} z_{r_{n}}+h_{n}$ and by Proposition $9, \phi_{n}$ has a unique decomposition $\phi_{n}=\phi_{n}^{1}+\cdots+\phi_{n}^{r_{n}}+H_{n}$ with $0 \leq \phi_{n}^{i} \leq r_{n} z_{i}$ for each $i$ and $0 \leq H_{n} \leq h_{n}$. The last inequality implies that $\lim _{n \rightarrow \infty} H_{n}=0$, because $\lim _{n \rightarrow \infty} h_{n}=0$ and the cone $X_{+}$is normal. Also we have $0 \leq \phi_{n}^{i} \leq u, r_{n} z_{i}$ for $i=1, \ldots, r_{n}$, therefore $\phi_{n}^{i}=a_{1}+\cdots+a_{r_{n}}+b_{n}$ with $0 \leq a_{j} \leq z_{j}$ for each $j$ and $0 \leq b_{n} \leq x_{r_{n}}$. Since the vectors $z_{1}, \ldots, z_{r_{n}}, x_{r_{n}}$ are pairwise disjoint in $X_{+}$we have $\phi_{n}^{i}=a_{i}$, therefore $0 \leq \phi_{n}^{i} \leq z_{i}$ for each $i=1, \ldots, r_{n}$. Since $H_{n} \leq u$, we have $H_{n}=\gamma_{1}+\cdots+\gamma_{r_{n}}+c_{n}$ with $0 \leq \gamma_{j} \leq z_{j}$ for each $j=1, \ldots, r_{n}$ and $0 \leq c_{n} \leq x_{r_{n}}$. Since $H_{n} \leq h_{n}$ we also have $\gamma_{j} \leq h_{n}$ for each $j$. Since the vectors $z_{j}, j=1, \ldots, r_{n}$, and $h_{n}$ are pairwise disjoint in $X_{+}$we have $\gamma_{j}=0$ for each $j=1, \ldots, r_{n}$, hence $H_{n}=c_{n}$, therefore $H_{n} \leq x_{r_{n}}$. So $\lim _{n \rightarrow \infty}\left(u-\left(\phi_{n}^{1}+\cdots+\phi_{n}^{r_{n}}+H_{n}\right)\right)=0$, therefore

$$
\lim _{n \rightarrow \infty}\left[\left(z_{1}-\phi_{n}^{1}\right)+\cdots+\left(z_{r_{n}}-\phi_{n}^{r_{n}}\right)+\left(x_{r_{n}}-H_{n}\right)\right]=0 .
$$

Since the members in the above limit are positive and the cone of $X_{+}$is normal we infer that $\lim _{n \rightarrow \infty}\left(x_{r_{n}}-H_{n}\right)=0$. We have shown above that $\lim H_{n}=0$, therefore $\lim x_{r_{n}}=0$. Since the sequence $\left\{x_{n}\right\}$ is decreasing it converges to zero and the proposition is proved.

Proposition 30. For each $x \in \delta(u)$ at least one successor of $x$ is an extremal point of $X_{+}$.

Proof. Let $x \in \delta(u)$. If at least one successor $x^{\prime}$ of $x$ does not belong to a branch of $\delta(u)$, then the process of decomposition stops after a finite number of steps at any successor of $x^{\prime}$, therefore any successor of $x^{\prime}$ is an extremal point of $X_{+}$dominated by $x$ and the assertion is proved. So suppose that any successor of $x$ belongs to a branch of $\delta(u)$. Also we may suppose that $x<u$ because in the case where $x=u$, it is enough to show the assertion for one of its successors.

Let $I$ be the closed solid subspace of $X$ generated by $x$ and set

$$
L=\left\{i \in \operatorname{supp}(x) \mid f_{i} \text { is not strictly positive on } I_{x}(X)\right\} .
$$

Then $L \subseteq M$. Also $\operatorname{supp}(x)=\operatorname{supp}\left(I_{+}\right)$. If $L$ is finite, then after a finite number of steps the decomposition stops at any successor of $x$ and the assertion holds. So suppose that $L$ is infinite. Let $j_{1}=\min L$. Then by Proposition 27(iii), there exists $x_{1} \in \delta(u)$ such that $x_{1} \leq x$ and $f_{j_{1}}$ is strictly positive on $\overline{I_{x_{1}}(X)}$. Since $x_{1}$ is an element of a branch of $\delta(u)$ dominated by $x$, and any such branch of $\delta(u)$ converges to zero, we may suppose that there exists $y_{1} \in \delta(u)$ such that $y_{1}<x_{1} \leq x$ and $\left\|y_{1}\right\| \leq 2^{-1} \varepsilon$, where $\varepsilon$ 
is a constant real number with $0<\varepsilon<\|x\|$. Note also that $f_{j_{1}}$ is strictly positive on $\overline{I_{y_{1}}(X)}$ because it is strictly positive on $\overline{I_{x_{1}}(X)}$ and $0<y_{1}<x_{1}$. By Proposition 27 we know that $\inf _{X_{+}}\left\{y_{1}, x-y_{1}\right\}=0$, hence $y_{1}$ is not a quasi-interior point of $I$. Therefore $\operatorname{supp}\left(y_{1}\right) \neq \operatorname{supp}\left(I_{+}\right)$, hence there exists $i \in \operatorname{supp}\left(I_{+}\right)$with $i \notin \operatorname{supp}\left(y_{1}\right)$, so there exists $i \in L$ with $y_{1}(i)=0$. We put $j_{2}=\min \left\{i \in L \mid y_{1}(i)=0\right\}$. Then $j_{1}<j_{2}$ and as before we can find a vector $y_{2} \in \delta(u)$ so that $y_{2}<x,\left\|y_{2}\right\| \leq 2^{-2} \varepsilon$ and $f_{j_{2}}$ is strictly positive on $\overline{I_{y_{2}}(X)}$. Then $\inf _{X_{+}}\left\{y_{1}, y_{2}\right\}=0$, because for any $h \in X$ with $0 \leq h \leq y_{1}, y_{2}$ we have $0 \leq h\left(j_{2}\right) \leq y_{1}\left(j_{2}\right)=0$, therefore $h=0$ because $f_{j_{2}}$ is strictly positive on $\overline{I_{y_{2}}(X)}$. In view of the method of selecting $y_{2}$ (as a sufficiently small member of a branch which converges to zero) we may also suppose that $y_{1} \in m_{\nu_{1}}$ and $y_{2} \in m_{\nu_{2}}$ with $\nu_{1}<\nu_{2}$. We may moreover suppose that $\nu_{2}$ is sufficiently large so that $m_{\nu_{2}}$, besides the successors of $x$ and the element $y_{2}$, contains at least one extra element so that

$$
m_{\nu_{2}}=\left\{y_{2}, a_{1}, \ldots, a_{k}, b_{1}, \ldots, b_{r}, c_{1}, \ldots, c_{l}\right\},
$$

where $a_{1}, \ldots, a_{k}$ are the successors of $y_{1}$ and $y_{2}, a_{1}, \ldots, a_{k}, b_{1}, \ldots, b_{r}$ are the successors of $x$. We put $s_{1}=y_{1}$ and $s_{2}=y_{1}+y_{2}$. Then $s_{1}<x$ and $s_{2}<x$. The first inequality is obvious and the second holds because $x$ is the sum of its successors in $m_{\nu_{2}}$. Also $s_{1}\left(j_{1}\right)>0$ and by the definition of $j_{2}$, we have $s_{2}(i)>0$ for each $i \in L$ with $i \leq j_{2}$. By Proposition 9 , $\inf _{X_{+}}\left\{s_{i}, x-s_{i}\right\}=0$ for each $i=1,2$, because the successors of $x$ in $m_{\nu_{2}}$ are pairwise disjoint. Since $\inf _{X_{+}}\left\{s_{2}, x-s_{2}\right\}=0$ we deduce that $s_{2}$ is not a quasi-interior point of $I_{+}$, hence there exists $i \in L$ with $s_{2}(i)=0$. Let $j_{3}=\min \left\{i \in L \mid s_{2}(i)=0\right\}$. Then $j_{2}<j_{3}$ and as before we can find $y_{3} \in m_{\nu_{3}}$ such that $\nu_{2}<\nu_{3},\left\|y_{3}\right\| \leq 2^{-3} \varepsilon, f_{j_{3}}$ is strictly positive on $\overline{I_{y_{3}}(X)}$ and the set of successors of $x$ in $m_{\nu_{3}}$ contains the successors of $y_{1}$, the successors of $y_{2}$, the element $y_{3}$ and at least one extra element. As before we can show that $\inf _{X_{+}}\left\{y_{1}, y_{3}\right\}=\inf _{X_{+}}\left\{y_{2}, y_{3}\right\}=0$. We put $s_{3}=s_{2}+y_{3}$.

Continuing this process we obtain a sequence $\left\{j_{\nu}\right\}$ in $L$ and sequences $\left\{y_{\nu}\right\},\left\{s_{\nu}\right\}$ in $X_{+}$such that $s_{1}=y_{1}, s_{\nu}=s_{\nu-1}+y_{\nu}$ for each $\nu=2,3, \ldots$, with the following properties:

(i) $0<s_{\nu}<s_{\nu+1}<x$,

(ii) $\left\|s_{\nu+1}-s_{\nu}\right\|=\left\|y_{\nu+1}\right\| \leq 2^{-\nu-1} \varepsilon$ and $y_{\nu} \in m_{k_{\nu}}$ with $k_{\nu}<k_{\nu+1}$ for each $\nu$,

(iii) $\inf _{X_{+}}\left\{s_{\nu}, x-s_{\nu}\right\}=0$ for each $\nu$,

(iv) $\left\{j_{\nu}\right\}$ is a strictly increasing sequence in $L$ and for each $i \in L$ with $i<j_{\nu+1}$ we have $s_{\nu}(i)>0$.

By (ii), $\left\{s_{\nu}\right\}$ is a Cauchy sequence; set $s=\lim _{\nu \rightarrow \infty} s_{\nu}$. Then $0 \leq s_{\nu} \leq s \leq x$ for each $\nu$. Since $\left\|s_{\nu}\right\| \leq \sum_{i=1}^{\nu}\left\|y_{i}\right\| \leq \varepsilon<\|x\|$, we have $s<x$. Also by (iv) 
and the fact that $\left\{s_{\nu}\right\}$ is increasing we see that $s(i)>0$ for each $i \in L$, therefore $\operatorname{supp}(s)=\operatorname{supp}\left(I_{+}\right)$. Hence $s$ is a quasi-interior point of $I_{+}$.

We will show that $\inf _{X_{+}}\{s, x-s\}=0$. To this end we suppose that $0 \leq h \leq s, x-s$. Since $s=s_{\nu}+\left(s-s_{\nu}\right)$ we have $h=h_{\nu}+h_{\nu}^{\prime}$ with $0 \leq h_{\nu} \leq s_{\nu}$ and $0 \leq h_{\nu}^{\prime} \leq\left(s-s_{\nu}\right)$. Since the cone is normal and $\lim \left(s-s_{\nu}\right)=0$ we have $\lim h_{\nu}^{\prime}=0$, therefore $h=\lim h_{\nu}$. Since $0 \leq h_{\nu} \leq s_{\nu}$ and $h_{\nu} \leq x-s \leq$ $x-s_{\nu}$ we infer that $h_{\nu}=0$ for each $\nu$, by (iii). Therefore $h=0$, hence $\inf _{X_{+}}\{s, x-s\}=0$. Since $I$ is solid we also obtain $\inf _{I_{+}}\{s, x-s\}=0$, which contradicts the fact that $s$ is a quasi-interior point of $I_{+}$(Proposition 5). Hence at least one successor $x^{\prime}$ of $x$ does not belong to a branch of $\delta(u)$, therefore at least one successor $x_{0}$ of $x$ is an extremal point of $X_{+}$and the proposition is proved.

Proposition 31. Any extremal point $x_{0}$ of $X_{+}$is a positive multiple of a unique element of $\delta(u)$.

Proof. By Proposition 5, since $x_{0}$ is an extremal point of $X_{+}$, there exists a real number $r>0$ with $r x_{0} \leq u$. Hence $r \leq a\|u\| /\left\|x_{0}\right\|$, where $a$ is the constant of the normal cone $X_{+}$. Therefore $\sup \left\{r \in \mathbb{R}_{+} \mid r x_{0} \leq u\right\}=\lambda>0$. Let $z_{0}=\lambda x_{0}$. Then $0<z_{0} \leq u$. Since $u=\sum_{z \in m_{\nu}} z$ and the elements of $m_{\nu}$ are pairwise disjoint, there exists a unique $y_{\nu} \in m_{\nu}$ so that $z_{0} \leq y_{\nu}$. Then $\inf _{X_{+}}\left\{z_{0}, x\right\}=0$ for each $x \in m_{\nu}, x \neq y_{\nu}$. Also $y_{\nu} \geq y_{\nu+1} \geq z_{0}$ for each $\nu$. Since each branch of $\delta(u)$ converges to zero, the process of decomposition stops at a point $y_{\mu}$ which is an extremal point of $X_{+}$with $z_{0} \leq y_{\mu}$. Hence $y_{\mu}=\lambda^{\prime} x_{0}$. Also $y_{\mu} \leq u$ and by the definition of $\lambda$ we have $\lambda^{\prime} \leq \lambda$, therefore $y_{\mu} \leq z_{0}$, which implies that $y_{\mu}=z_{0}$ and $z_{0} \in \delta(u)$. If $z_{0}^{\prime}=k x_{0} \in \delta(u)$, then $k x_{0} \leq u$, therefore $k \leq \lambda$ and $z_{0}^{\prime} \leq z_{0}$. Hence $z_{0}^{\prime}$ is a successor of $z_{0}$. If $z_{0}^{\prime}<z_{0}$ we get a contradiction because $z_{0}$, being an extremal point of $X_{+}$, is indecomposable. Therefore $z_{0}^{\prime}=z_{0}$ and the proposition is proved.

In our main result below we prove that $X$ has a positive basis. This basis is also unconditional because $X_{+}$is generating and normal. For convenience we repeat the standing assumptions on $E$ and $X$.

THEOREM 32. Let $E$ be an ordered Banach space and suppose that $E_{+}$ is defined by the family $\mathcal{F}=\left\{f_{i} \mid i \in \mathbb{N}\right\} \subset E_{+}^{*}$. Let $X$ be a closed ordered subspace of $E$ with the Riesz decomposition property and suppose that $X_{+}$ is normal and generating. If $X$ has the maximum support property and the ws-property with respect to $\mathcal{F}$, then $X$ has a positive basis.

Proof. Let $B$ be the set of extremal points of $X_{+}$with norm 1. By Proposition $30, B \neq \emptyset$, and by the previous proposition the map $T: B \rightarrow \delta(u)$ so that $T(x)=\lambda x \in \delta(u)$ is one-to-one. Since $\delta(u)$ is countable, so is $B$, say $B=\left\{u_{i}: i \in \mathbb{N}\right\}$ and $b_{i}=\lambda_{i} u_{i} \in \delta(u)$. Let $u_{0}=\sum_{i=1}^{\infty} b_{i} / 2^{i}$. For each 
$i \in M$ there exists $z \in \delta(u)$ so that $f_{i}$ is strictly positive on $I=\overline{I_{z}(X)}$. By Proposition 30, $z \geq b_{j}$ for at least one $j$, therefore $b_{j}(i)>0$. Hence $\operatorname{supp}\left(u_{0}\right)=\operatorname{supp}\left(X_{+}\right)$and $u_{0}$ is a quasi-interior point of $X_{+}$.

Let $x \in X_{+}$. Then there exists an increasing sequence $x_{n} \in[0, x] \cap$ $\left[0, k_{n} u_{0}\right]$ where the sequence $k_{n}$ is strictly increasing and $\lim _{n \rightarrow \infty} x_{n}=x$. Since $0 \leq x_{n} \leq k_{n} u_{0}$, each $x_{n}$ has a unique expansion $x_{n}=\sum_{i=1}^{\infty} \sigma_{n i} u_{i}$ with $\sigma_{n i} \in \mathbb{R}_{+}$, by Proposition 9. The sequence $\left\{\sigma_{n i} \mid n \in \mathbb{N}\right\}$ is increasing. Indeed, for $m>n$ we take again the expansion $x_{m}-x_{n}=$ $\sum_{i=1}^{\infty} a_{i} u_{i}$ and we have $\sigma_{m i}=\sigma_{n i}+a_{i} \geq \sigma_{n i}$. Set $\sigma_{i}=\lim _{n \rightarrow \infty} \sigma_{n i}$. Then $0 \leq \sigma_{i} u_{i} \leq x$, because $0 \leq \sigma_{n i} u_{i} \leq x$ for each $i$. For each $m \in \mathbb{N}$ we have $\sum_{i=1}^{m} \sigma_{n i} u_{i} \leq x_{n} \leq x$ and by taking limits as $n \rightarrow \infty$ we see that $\sum_{i=1}^{m} \sigma_{i} u_{i} \leq x$. Since $\left\{x_{n}\right\}$ converges to $x$ there exists a strictly increasing sequence $m_{n}$ of natural numbers so that the sequence $y_{n}=\sum_{i=1}^{m_{n}} \sigma_{n i} u_{i}$ converges to $x$. Then $\sum_{i=1}^{m_{n}} \sigma_{n i} u_{i} \leq \sum_{i=1}^{m_{n}} \sigma_{i} u_{i} \leq x$, which yields $x=\sum_{i=1}^{\infty} \sigma_{i} u_{i}$. Let $\bar{u}_{j}=\sum_{i \neq j} b_{i} / 2^{i}$. Then $\bar{u}_{j}$ is not a quasi-interior point of $X_{+}$, because $\inf _{X_{+}}\left\{b_{j}, \bar{u}_{j}\right\}=0$ by Proposition 9 . Therefore $\operatorname{supp}\left(\bar{u}_{j}\right)$ is a proper subset of $\operatorname{supp}\left(X_{+}\right)$, hence there exists $k_{j} \in M$ with $f_{k_{j}}\left(\bar{u}_{j}\right)=0$. Hence $f_{k_{j}}\left(u_{i}\right)=0$ for each $i \neq j$. Also $f_{k_{j}}\left(u_{j}\right)>0$ because $f_{k_{j}}\left(u_{0}\right)>0$. Let $g_{j}=f_{k_{j}} / f_{k_{j}}\left(u_{j}\right)$. Then for each $x \in X_{+}$we have $g_{j}(x)=\sigma_{j}$, therefore $x=\sum_{i=1}^{\infty} g_{i}(x) u_{i}$. Since the cone $X_{+}$is generating we conclude that $x=\sum_{i=1}^{\infty} g_{i}(x) u_{i}$ for each $x \in X$ and this expansion is unique. Therefore $\left\{u_{n}\right\}$ is a positive basis of $X$.

By the previous result and Corollaries 20 and 21 we have:

Corollary 33. Let $E$ be a Banach lattice with order continuous norm and suppose that $E_{+}$is defined by a countable family $\mathcal{F} \subset E_{+}^{*}$. Let $X$ be a closed ordered subspace of $E$ with the Riesz decomposition property and generating positive cone $X_{+}$. If $X$ has the maximum support property with respect to $\mathcal{F}$, then $X$ has a positive basis.

Corollary 34. Let $E$ be an ordered Banach space whose positive cone is defined by a family $\mathcal{F}=\left\{f_{i} \mid i \in \mathbb{N}\right\} \subset E_{+}^{*}$. Suppose also that $E$ is a dual space and that the functionals $f_{i}$ are weak-star continuous. If $X$ is a closed ordered subspace of $E$ with the Riesz decomposition property, and $X_{+}$is weak-star closed, normal and generating, and $X$ has the maximum support property with respect to $\mathcal{F}$, then $X$ has a positive basis.

REMARK 35. In the special case where $E=\ell_{\infty}$ and $X$ is a weak-star closed ordered subspace of $\ell_{\infty}$ with the RDP and generating positive cone $X_{+}$we have: If $X$ has the maximum support property with respect to the family of Dirac measures supported at natural numbers, then $X$ has a positive basis. 
4. Biorthogonal systems. The results of the previous section can be applied to the problem: under what conditions does a biorthogonal system define a positive basis? So in this section we suppose that $E$ is an ordered Banach space with a positive biorthogonal system $\left\{\left(e_{i}, f_{i}\right) \mid i \in \mathbb{N}\right\}$, i.e. $e_{i} \in E$ and $f_{i} \in E_{+}^{*}$ for each $i, f_{i}\left(e_{i}\right)=1, f_{i}\left(e_{j}\right)=0$ for all $j \neq i$, and the family $\mathcal{F}=\left\{f_{i} \mid i \in \mathbb{N}\right\}$ defines the positive cone of $E$. In the next results the positive basis of $E$ is also unconditional.

THEOREM 36. Let $E$ be an ordered Banach space with a positive biorthogonal system $\left\{\left(e_{i}, f_{i}\right) \mid i \in \mathbb{N}\right\}$. If $E_{+}$is normal and generating and $E$ has the Riesz decomposition property, then the following statements are equivalent:

(i) The sequence $\left\{e_{i}\right\}$ of the biorthogonal system is a positive basis of $E$,

(ii) $E$ has the maximum support property and the ws-property with respect to the family $\mathcal{F}=\left\{f_{i} \mid i \in \mathbb{N}\right\}$.

Proof. Suppose that $\left\{e_{i}\right\}$ is a positive basis of $E$. Since $\left\{\left(e_{i}, f_{i}\right)\right\}$ is a positive biorthogonal system of $E$ we know that $f_{i}\left(e_{i}\right)=1$ and $f_{i}\left(e_{j}\right)=0$ for all $j \neq i$; therefore, by Theorem 16, $E$ has the maximum support property with respect to $\mathcal{F}$. Since $\left\{e_{i}\right\}$ is a positive basis of $E$, by Corollary $22, E$ has the ws-property, so (i) implies (ii). Suppose now that (ii) holds. Then $E$ has a positive basis $\left\{b_{n}\right\}$. Since $E$ has the maximum support property with respect to $\mathcal{F}, E$ has the minimal support property, therefore an element $x_{0}$ of $E_{+}$is an extremal point of $E_{+}$if and only if $x_{0}$ has minimal support in $E_{+}$. Therefore the extremal points of $E_{+}$are the positive multiples of the elements $e_{n}\left(\operatorname{supp}\left(e_{n}\right)=\{n\}\right)$. Since the elements of the positive basis define the extremal rays of $E_{+}$it follows that the basis $\left\{b_{n}\right\}$ coincides, up to a scalar multiple and proper enumeration, with the sequence $\left\{e_{n}\right\}$.

Corollary 37. Let $E$ be an ordered Banach space with a positive biorthogonal system $\left\{\left(e_{i}, f_{i}\right) \mid i \in \mathbb{N}\right\}$ and suppose that $E$ has the Riesz decomposition property and either

(a) $E$ is a Banach lattice with order continuous norm, or

(b) $E$ is a dual space, the positive cone $E_{+}$of $E$ is weak-star closed, normal and generating, and the functionals $f_{i}$ are weak-star continuous.

Then the following statements are equivalent:

(i) the sequence $\left\{e_{i}\right\}$ of the biorthogonal system is a positive basis of $E$,

(ii) $E$ has the maximum support property with respect to the family $\left\{f_{i} \mid i \in \mathbb{N}\right\}$.

REMARK 38. According to Corollary 37, the sequence $\left\{e_{i}\right\}$ of the usual biorthogonal system $\left\{e_{i}, \delta_{i}\right\}$ of $\ell_{\infty}$ is not a positive basis of $\ell_{\infty}$ because it does not have the maximum support property with respect to the family $\left\{\delta_{i}\right\}$ (Example 15). 


\section{References}

[1] C. D. Aliprantis and O. Burkinshaw, Positive Operators, Academic Press, New York, 1985.

[2] N. Danet, The Riesz decomposition property for the space of regular operators, Proc. Amer. Math. Soc. 129 (2000), 539-542.

[3] W. T. Gowers and B. Maurey, The unconditional basic sequence problem, J. Amer. Math. Soc. 6 (1993), 851-874.

[4] G. J. O. Jameson, Ordered Linear Spaces, Lecture Notes in Math. 141, Springer, Heidelberg, 1970.

[5] M. A. Krasnosel'skij, Je. A. Lifshits, and A. V. Sobolev, Positive Linear SystemsThe Method of Positive Operators, Sigma Ser. Appl. Math. 5, Heldermann, 1989.

[6] P. Meyer-Nierberg, Banach Lattices, Springer, 1991.

[7] I. A. Polyrakis, Schauder bases in locally solid lattice Banach spaces, Math. Proc. Cambridge Philos. Soc. 101 (1987), 91-105.

[8] —, Lattice-subspaces of $C[0,1]$ and positive bases, J. Math. Anal. Appl. 184 (1994), $1-15$.

[9] I. Singer, Bases in Banach Spaces - I, Springer, Heidelberg, 1970.

[10] B. Z. Vulikh, Introduction to the Theory of Cones in Normed Spaces, Kalinin, 1977 (in Russian).

[11] A. W. Wickstead, Compact subsets of partially ordered Banach spaces, Math. Ann. 212 (1975), 271-284.

Department of Mathematics

National Technical University of Athens

Zografou Campus 157 80, Athens, Greece

E-mail: ypoly@math.ntua.gr

Received July 27, 2004

Revised version February 10, 2006 